\title{
Controllability Criteria for Linear Fractional Differential Systems with State Delay and Impulses
}

\author{
Hai Zhang, ${ }^{1,2}$ Jinde Cao, ${ }^{1,3}$ and Wei Jiang ${ }^{4}$ \\ ${ }^{1}$ Department of Mathematics, Southeast University, Nanjing, Jiangsu 210096, China \\ ${ }^{2}$ Department of Mathematics, Anqing Normal University, Anqing, Anhui 246133, China \\ ${ }^{3}$ Department of Mathematics, King Abdulaziz University, Jeddah 21589, Saudi Arabia \\ ${ }^{4}$ School of Mathematical Sciences, Anhui University, Hefei, Anhui 230039, China
}

Correspondence should be addressed to Jinde Cao; jdcao@seu.edu.cn

Received 8 April 2013; Accepted 14 May 2013

Academic Editor: Francisco J. Marcellán

Copyright (C) 2013 Hai Zhang et al. This is an open access article distributed under the Creative Commons Attribution License, which permits unrestricted use, distribution, and reproduction in any medium, provided the original work is properly cited.

\begin{abstract}
This paper is concerned with the controllability of linear fractional differential systems with delay in state and impulses. The factors of such systems including fractional derivative, impulses, and delay are taken into account synchronously. The expression of state response for such systems is derived, and the sufficient and necessary conditions of controllability criteria are established. Both the proposed criteria and illustrative examples show that the controllability property of the linear systems is dependent neither on the order of fractional derivative, on delay nor on impulses.
\end{abstract}

\section{Introduction}

In this paper, we consider the controllability of linear fractional differential systems with state delay and impulses as follows:

$$
\begin{gathered}
D^{\alpha} x(t)=A x(t)+B x(t-\tau)+C u(t), \\
t \in[0, T] \backslash\left\{t_{1}, t_{2}, \ldots, t_{k}\right\}, \\
\Delta x\left(t_{i}\right)=x\left(t_{i}^{+}\right)-x\left(t_{i}^{-}\right)=I_{i}\left(x\left(t_{i}\right)\right), \quad i=1,2, \ldots, k, \\
\quad x(t)=\varphi(t), \quad t \in[-\tau, 0],
\end{gathered}
$$

where $D^{\alpha} x(t)$ denotes an $\alpha$ order Caputo's fractional derivative of $x(t), 0<\alpha<1, A, B$, and $C$ are the known constant matrices and satisfy $A, B \in \mathbb{R}^{n \times n}, C \in \mathbb{R}^{n \times m}, \tau$ is a positive constant, $x \in \mathbb{R}^{n}$ is the state variable, $u \in \mathbb{R}^{m}$ is the control input, $\varphi \in \mathbf{C}\left([-\tau, 0], \mathbb{R}^{n}\right)$ is the initial state function, where $\mathbf{C}\left([-\tau, 0], \mathbb{R}^{n}\right)$ denotes the space of all continuous functions mapping the interval $[-\tau, 0]$ into $\mathbb{R}^{n}, I_{i}: \mathbb{R}^{n} \longrightarrow \mathbb{R}^{n}$ is continuous for $i=1,2, \ldots, k$, and

$$
x\left(t_{i}^{+}\right)=\lim _{\varepsilon \rightarrow 0^{+}} x\left(t_{i}+\varepsilon\right), \quad x\left(t_{i}^{-}\right)=\lim _{\varepsilon \rightarrow 0^{-}} x\left(t_{i}+\varepsilon\right)
$$

represent the right and left limits of $x(t)$ at $t=t_{i}$ and the discontinuous points

$$
t_{1}<t_{2}<\cdots<t_{i}<\cdots<t_{k},
$$

where $0=t_{0}<\tau<t_{1}, t_{k}<t_{k+1}=T<+\infty$, and $x\left(t_{i}\right)=x\left(t_{i}^{-}\right)$ which implies that the solution of system (1) is left continuous at $t_{i}$.

The subject of fractional differential equations is gaining much importance and attention (see [1-11] and references therein). Fractional differential equations have been proved to be an excellent tool in the modelling of many phenomena in various fields of engineering, physics, and economics. In fact, fractional differential equations are considered as an alternative model to nonlinear differential equations. At the same time, time delay is one of the inevitable problems in practical engineering applications, which has an important effect on the stability and performance of system. In the last few years, the results with regard to the fractional delay differential systems have been presented in [12-15].

Although most dynamical systems are analyzed in either the continuous or discrete-time domain, many real systems 
in physics, chemistry, biology, engineering, and information science may experience abrupt changes as certain instants during the continuous dynamical processes. This kind of impulsive behaviors can be modeled by impulsive systems. The basic theory of impulsive differential equations can be found in the monographs of Bainov and Simeonov [16], Benchohra et al. [17], and the paper of Fečkan et al. [18].

On the other hand, controllability is the most fundamental concept in modern control theory, which has close connections to pole assignment, structural decomposition, quadratic optimal control, and so forth. Some important results concerning the control theory for various kinds of systems have been obtained in [19-36] and references therein. Kalman et al. [19] have investigated the controllability of linear dynamical systems based on the algebraic approach. Wonham and Morse [20] have discussed the pole assignment problems of linear systems based on the geometric approach. In [21-24], the authors have discussed the controllability of integer derivative delay systems. In $[25,26]$, the controllability of the descriptor (singular) systems has been considered. Impulsive control systems with integer derivative have been investigated in [27-29]. For integer derivative control systems with state delay and impulses, Zhang et al. [27] have derived the sufficient conditions for the controllability based on the fixed point theorem. It is worth pointing out that notable contributions have been made to fractional control systems in [30-36]. The different techniques have been developed to investigate the control problems of fractional differential systems, such as fractional sliding manifold approach [30], fixed point theorems [31-34], functional analysis method [33, 34], and algebraic method $[35,36]$. To the best of our knowledge, there are no relevant reports on the controllability of fractional differential systems with state delay and impulses as treated in the current literature. In this paper, the factors of control systems including the Caputo's fractional derivative, impulses, and delay are taken into account synchronously. The purpose of this paper is to establish the sufficient and necessary conditions of controllability for system (1) based on the algebraic approach. The recent research surge in developing the theory of fractional control systems has motivated and inspired our present work.

This paper is organized as follows. In Section 2, we recall some definitions and preliminary facts, and the expression of state response for system (1) is derived. In Section 3, the sufficient and necessary conditions of controllability criteria are established. In Section 4, some examples are given to illustrate the effectiveness and applicability of controllability criteria. Finally, some concluding remarks are drawn in Section 5 .

\section{Preliminaries}

Throughout this paper, denote by $\mathbf{C}_{p}\left([0, T], \mathbb{R}^{n}\right)$ the space of all piecewise left continuous functions mapping the interval $[0, T]$ into $\mathbb{R}^{n}$.

Let us recall some definitions and preliminary facts. For more details, one can see [1-4].
Definition 1. The Riemann-Liouville's fractional integral of order $\alpha>0$ with the lower limit zero for a function $f: R^{+} \rightarrow$ $R^{n}$ is defined as

$$
D^{-\alpha} f(t)=\frac{1}{\Gamma(\alpha)} \int_{0}^{t}(t-s)^{\alpha-1} f(s) d s, \quad t>0,
$$

provided the right side is pointwise defined on $[0,+\infty)$, where $\Gamma(\cdot)$ is the Gamma function.

Definition 2. The Caputo's fractional derivative of order $\alpha$ for a function $f: R^{+} \rightarrow R^{n}$ is defined as

$$
\begin{array}{r}
D^{\alpha} f(t)=\frac{1}{\Gamma(m-\alpha+1)} \int_{0}^{t}(t-s)^{m-\alpha} f^{(m+1)}(s) d s, \\
0 \leq m \leq \alpha<m+1 .
\end{array}
$$

Definition 3. The Mittag-Leffler function in two parameters is defined as

$$
E_{\alpha, \beta}(z)=\sum_{k=0}^{+\infty} \frac{z^{k}}{\Gamma(k \alpha+\beta)},
$$

where $\alpha>0, \beta>0$, and $z \in \mathbb{C}, \mathbb{C}$ denotes the complex plane.

Definition 4. The Laplace transform of a function $f(t)$ is defined as

$$
F(s)=\mathfrak{E}[f(t)]=\int_{0}^{+\infty} e^{-s t} f(t) d t, \quad s \in \mathbb{C},
$$

where $f(t)$ is $n$-dimensional vector-valued function.

Remark 5. If $\alpha \in(0,1)$, then

$$
\mathfrak{E}\left[\left(D^{\alpha} f\right)(t)\right]=s^{\alpha} \mathfrak{E}[f(t)]-s^{\alpha-1} f(0) .
$$

Lemma 6 (see [2]). Let $\mathbb{C}$ be complex plane, for any $\alpha>0$, $\beta>0$, and $A \in \mathbb{C}^{n \times n}$; then

$$
£\left[t^{\beta-1} E_{\alpha, \beta}\left(A t^{\alpha}\right)\right]=s^{\alpha-\beta}\left(s^{\alpha} I-A\right)^{-1}, \quad \Re(s)>\|A\|^{1 / \alpha}
$$

holds, where $\mathfrak{R}(s)$ represents the real part of the complex number $s$ and I denotes the identity matrix.

In order to obtain the state response of system (1), we firstly consider the representation of solution for linear fractional delay differential systems without impulses as follows:

$$
\begin{gathered}
D^{\alpha} x(t)=A x(t)+B x(t-\tau)+f(t), \quad t \in[0, T], \\
x(t)=\varphi(t), \quad t \in[-\tau, 0] .
\end{gathered}
$$

Lemma 7. Let $0<\alpha<1$; if $f:[0, T] \rightarrow \mathbb{R}^{n}$ is continuous and exponentially bounded, then the solution of system (10) can be represented as

$$
\begin{aligned}
x(t)=\varphi(0)+\int_{0}^{t}(t-s)^{\alpha-1} E_{\alpha, \alpha}\left[A(t-s)^{\alpha}\right] & \\
& \times[A \varphi(0)+B x(s-\tau)+f(s)] d s, \\
& t \in[0, T]
\end{aligned}
$$

and $x(t)=\varphi(t), t \in[-\tau, 0]$. 
Proof. Applying the method of steps which has been presented in [12], then there exists a unique solution to system (10).

For $t \in[0, T]$, taking the Laplace transform with respect to $t$ in both sides of system (10), we obtain

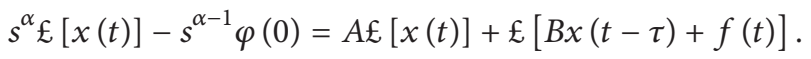

Then (12) can be written as

$$
\begin{aligned}
£[x(t)]= & \left(s^{\alpha} I-A\right)^{-1} s^{\alpha-1} \varphi(0) \\
& +\left(s^{\alpha} I-A\right)^{-1} £[B x(t-\tau)+f(t)] .
\end{aligned}
$$

From Definition 4 and Lemma 6, then (13) is equivalent to

$$
\begin{aligned}
£[x(t)]= & \left(s^{\alpha} I-A\right)^{-1} s^{\alpha-1} \varphi(0) \\
& +\left(s^{\alpha} I-A\right)^{-1} £[B x(t-\tau)+f(t)] \\
= & \left(s^{\alpha} I-A\right)^{-1} s^{\alpha} £[\varphi(0)] \\
& +\left(s^{\alpha} I-A\right)^{-1} £[B x(t-\tau)+f(t)] \\
= & £[\varphi(0)]+\left(s^{\alpha} I-A\right)^{-1} \\
& \times £[A \varphi(0)+B x(t-\tau)+f(t)] \\
= & £[\varphi(0)]+£\left[t^{\alpha-1} E_{\alpha, \alpha}\left(A t^{\alpha}\right)\right] \\
& \times £[A \varphi(0)+B x(t-\tau)+f(t)] .
\end{aligned}
$$

The convolution theorem of the Laplace transform applied to (14) yields the form

$$
\begin{aligned}
£[x(t)]= & £[\varphi(0)] \\
+ & £\left\{\int_{0}^{t}(t-s)^{\alpha-1} E_{\alpha, \alpha}\left[A(t-s)^{\alpha}\right]\right. \\
& \quad \times[A \varphi(0)+B x(s-\tau)+f(s)] d s\} .
\end{aligned}
$$

Applying the inverse Laplace transform, we obtain

$$
\begin{array}{rl}
x(t)=\varphi(0)+\int_{0}^{t}(t-s)^{\alpha-1} E_{\alpha, \alpha}\left[A(t-s)^{\alpha}\right] & \\
& \times[A \varphi(0)+B x(s-\tau)+f(s)] d s, \\
t & t \in[0, T] .
\end{array}
$$

Therefore, we have the stated result.

Lemma 8. Let $0<\alpha<1$ and $u \in \mathbf{C}_{p}\left([0, T], \mathbb{R}^{m}\right)$; then state response of system (1) can be represented as follows.

For $t \in[-\tau, 0]$,

$$
x(t)=\varphi(t)
$$

For $t \in\left[0, t_{1}\right]$

$$
\begin{aligned}
x(t)=\varphi(0)+\int_{0}^{t}(t-s)^{\alpha-1} E_{\alpha, \alpha}\left[A(t-s)^{\alpha}\right] \\
\quad \times[A \varphi(0)+B x(s-\tau)+C u(s)] d s .
\end{aligned}
$$

For $t \in\left(t_{1}, t_{2}\right]$,

$$
\begin{aligned}
& x(t)=\varphi(0)+I_{1}\left(x\left(t_{1}^{-}\right)\right) \\
& +\int_{0}^{t}(t-s)^{\alpha-1} E_{\alpha, \alpha}\left[A(t-s)^{\alpha}\right] \\
& \times[A \varphi(0)+B x(s-\tau)+C u(s)] d s . \\
& \text { For } t \in\left(t_{i}, t_{i+1}\right], i=1,2, \ldots, k \text {, } \\
& x(t)=\varphi(0)+\sum_{j=1}^{i} I_{j}\left(x\left(t_{j}^{-}\right)\right) \\
& +\int_{0}^{t}(t-s)^{\alpha-1} E_{\alpha, \alpha}\left[A(t-s)^{\alpha}\right] \\
& \times[A \varphi(0)+B x(s-\tau)+C u(s)] d s .
\end{aligned}
$$

Proof. If $t \in[-\tau, 0]$, then the conclusion obviously holds. If $t \in\left[0, t_{1}\right]$, then, from Lemma 7 ,

$$
\begin{aligned}
& x(t)=\varphi(0)+\int_{0}^{t}(t-s)^{\alpha-1} E_{\alpha, \alpha}\left[A(t-s)^{\alpha}\right] \\
& \times[A \varphi(0)+B x(s-\tau)+C u(s)] d s, \\
& x\left(t_{1}\right)=\varphi(0)+\int_{0}^{t_{1}}\left(t_{1}-s\right)^{\alpha-1} E_{\alpha, \alpha}\left[A\left(t_{1}-s\right)^{\alpha}\right] \\
& \quad \times[A \varphi(0)+B x(s-\tau)+C u(s)] d s .
\end{aligned}
$$

If $t \in\left(t_{1}, t_{2}\right]$, applying the idea used in [18], we have

$$
\begin{aligned}
x(t)= & x\left(t_{1}^{+}\right) \\
& -\int_{0}^{t_{1}}\left(t_{1}-s\right)^{\alpha-1} E_{\alpha, \alpha}\left[A\left(t_{1}-s\right)^{\alpha}\right] \\
& \times[A \varphi(0)+B x(s-\tau)+C u(s)] d s \\
& +\int_{0}^{t}(t-s)^{\alpha-1} E_{\alpha, \alpha}\left[A(t-s)^{\alpha}\right] \\
& \quad \times[A \varphi(0)+B x(s-\tau)+C u(s)] d s
\end{aligned}
$$




$$
\begin{aligned}
& =x\left(t_{1}^{-}\right)+I_{1}\left(x\left(t_{1}^{-}\right)\right) \\
& -\int_{0}^{t_{1}}\left(t_{1}-s\right)^{\alpha-1} E_{\alpha, \alpha}\left[A\left(t_{1}-s\right)^{\alpha}\right] \\
& \quad \times[A \varphi(0)+B x(s-\tau)+C u(s)] d s \\
& +\int_{0}^{t}(t-s)^{\alpha-1} E_{\alpha, \alpha}\left[A(t-s)^{\alpha}\right] \\
& \quad \times[A \varphi(0)+B x(s-\tau)+C u(s)] d s \\
& =\varphi(0)+I_{1}\left(x\left(t_{1}^{-}\right)\right) \\
& +\int_{0}^{t}(t-s)^{\alpha-1} E_{\alpha, \alpha}\left[A(t-s)^{\alpha}\right] \\
& \times[A \varphi(0)+B x(s-\tau)+C u(s)] d s .
\end{aligned}
$$

If $t \in\left(t_{2}, t_{3}\right]$, then

$$
\begin{aligned}
x(t)= & x\left(t_{2}^{+}\right)-\int_{0}^{t_{2}}\left(t_{2}-s\right)^{\alpha-1} E_{\alpha, \alpha}\left[A\left(t_{2}-s\right)^{\alpha}\right] \\
& \times[A \varphi(0)+B x(s-\tau)+C u(s)] d s \\
& +\int_{0}^{t}(t-s)^{\alpha-1} E_{\alpha, \alpha}\left[A(t-s)^{\alpha}\right] \\
& \times[A \varphi(0)+B x(s-\tau)+C u(s)] d s \\
= & x\left(t_{2}^{-}\right)+I_{2}\left(x\left(t_{2}^{-}\right)\right) \\
& -\int_{0}^{t_{2}}\left(t_{2}-s\right)^{\alpha-1} E_{\alpha, \alpha}\left[A\left(t_{2}-s\right)^{\alpha}\right] \\
& \times[A \varphi(0)+B x(s-\tau)+C u(s)] d s \\
& +\int_{0}^{t}(t-s)^{\alpha-1} E_{\alpha, \alpha}\left[A(t-s)^{\alpha}\right] \\
& \times[A \varphi(0)+B x(s-\tau)+C u(s)] d s \\
= & \varphi(0)+\sum_{j=1}^{2} I_{j}\left(x\left(t_{j}^{-}\right)\right) \\
& +\int_{0}^{t}(t-s)^{\alpha-1} E_{\alpha, \alpha}\left[A(t-s)^{\alpha}\right] \\
& \times[A \varphi(0)+B x(s-\tau)+C u(s)] d s .
\end{aligned}
$$

If $t \in\left(t_{i}, t_{i+1}\right](i=1,2, \ldots, k)$, then the same argument implies the following expression:

$$
\begin{aligned}
x(t)= & \varphi(0)+\sum_{j=1}^{i} I_{j}\left(x\left(t_{j}^{-}\right)\right) \\
& +\int_{0}^{t}(t-s)^{\alpha-1} E_{\alpha, \alpha}\left[A(t-s)^{\alpha}\right] \\
& \quad \times[A \varphi(0)+B x(s-\tau)+C u(s)] d s .
\end{aligned}
$$

Thus, the proof is completed.

\section{Controllability Criteria for System (1)}

In this section, we establish the sufficient and necessary conditions of controllability criteria for system (1) based on the algebraic approach.

Definition 9. System (1) is called controllable on $[0, \omega](\omega \in$ $(0, T])$; for any initial function $\varphi \in \mathbf{C}\left([-\tau, 0], \mathbb{R}^{n}\right)$ and any state $x_{\omega} \in \mathbb{R}^{n}$, there exists a control input $u(t) \in \mathbf{C}_{p}([0, \omega]$, $\left.\mathbb{R}^{m}\right)$, such that the corresponding solution of (1) satisfies $x(\omega)=x_{\omega}$.

Theorem 10. System (1) is controllable on $[0, \omega]$ if and only if the Gramian matrix

$$
\begin{aligned}
W_{c}[0, \omega]= & \int_{0}^{\omega}(\omega-s)^{\alpha-1}\left[E_{\alpha, \alpha}\left(A(\omega-s)^{\alpha}\right)\right] \\
& \times C C^{*}\left[E_{\alpha, \alpha}\left(A^{*}(\omega-s)^{\alpha}\right)\right] d s
\end{aligned}
$$

is nonsingular for some $\omega \in[0, T]$, where $E_{\alpha, \alpha}(\cdot)$ is the MittagLeffler function and $*$ denotes the matrix transpose.

Proof. We firstly prove sufficiency of Theorem 10. If $W_{c}[0, \omega]$ is nonsingular, then $W_{c}^{-1}[0, \omega]$ is well defined. For any initial state $\varphi \in \mathbf{C}\left([-\tau, 0], \mathbb{R}^{n}\right)$, when $\omega \in\left[0, t_{1}\right]$, we take the control function as

$$
\begin{aligned}
u(t)= & C^{*}\left[E_{\alpha, \alpha}\left(A^{*}(\omega-t)^{\alpha}\right)\right] W_{c}^{-1}[0, \omega] \\
& \times\left[x_{\omega}-\varphi(0)-\int_{0}^{\omega}(\omega-\theta)^{\alpha-1} E_{\alpha, \alpha}\left(A(\omega-\theta)^{\alpha}\right)\right. \\
& \times(A \varphi(0)+B x(\theta-\tau)) d \theta] .
\end{aligned}
$$

Substituting $t=\omega$ in (18) and inserting (26) yield

$$
\begin{aligned}
x(\omega)=\varphi(0)+\int_{0}^{\omega}(\omega-s)^{\alpha-1} E_{\alpha, \alpha}\left[A(\omega-s)^{\alpha}\right] & \\
\times & \{A \varphi(0)+B x(s-\tau) \\
& +C C^{*}\left[E_{\alpha, \alpha}\left(A^{*}(\omega-s)^{\alpha}\right)\right] \\
& \times W_{c}^{-1}[0, \omega] \\
& \times\left[x_{\omega}-\varphi(0)\right. \\
-\int_{0}^{\omega}(\omega-\theta)^{\alpha-1} & \times E_{\alpha, \alpha}\left(A(\omega-\theta)^{\alpha}\right) \\
\times(A \varphi(0) & +B x(\theta-\tau)) d \theta]\} d s
\end{aligned}
$$




$$
\begin{aligned}
& =\varphi(0)+\int_{0}^{\omega}(\omega-s)^{\alpha-1} E_{\alpha, \alpha}\left[A(\omega-s)^{\alpha}\right] \\
& \quad \times[A \varphi(0)+B x(s-\tau)] d s \\
& +\left[x_{\omega}-\varphi(0)-\int_{0}^{\omega}(\omega-\theta)^{\alpha-1} E_{\alpha, \alpha}\left(A(\omega-\theta)^{\alpha}\right)\right. \\
& \times(A \varphi(0)+B x(\theta-\tau)) d \theta] \\
& =x_{\omega} .
\end{aligned}
$$

Thus system (1) is controllable on $[0, \omega], \omega \in\left[0, t_{1}\right]$.

For $\omega \in\left(t_{1}, t_{2}\right]$, we take the control function as

$$
\begin{aligned}
u(t)= & C^{*}\left[E_{\alpha, \alpha}\left(A^{*}(\omega-t)^{\alpha}\right)\right] W_{c}^{-1}[0, \omega] \\
& \times\left[x_{\omega}-\varphi(0)-I_{1}\left(x\left(t_{1}^{-}\right)\right)\right. \\
& -\int_{0}^{\omega}(\omega-\theta)^{\alpha-1} E_{\alpha, \alpha}\left(A(\omega-\theta)^{\alpha}\right) \\
& \quad \times(A \varphi(0)+B x(\theta-\tau)) d \theta] .
\end{aligned}
$$

Substituting $t=\omega$ in (19) and inserting (28) yield

$$
\begin{aligned}
& x(\omega)=\varphi(0)+I_{1}\left(x\left(t_{1}^{-}\right)\right) \\
& +\int_{0}^{\omega}(\omega-s)^{\alpha-1} E_{\alpha, \alpha}\left[A(\omega-s)^{\alpha}\right] \\
& \times\{A \varphi(0)+B x(s-\tau) \\
& +C C^{*}\left[E_{\alpha, \alpha}\left(A^{*}(\omega-s)^{\alpha}\right)\right] W_{c}^{-1}[0, \omega] \\
& \times\left[x_{\omega}-\varphi(0)-I_{1}\left(x\left(t_{1}^{-}\right)\right)\right. \\
& -\int_{0}^{\omega}(\omega-\theta)^{\alpha-1} E_{\alpha, \alpha}\left(A(\omega-\theta)^{\alpha}\right) \\
& \times(A \varphi(0)+B x(\theta-\tau)) d \theta]\} d s \\
& =\varphi(0)+I_{1}\left(x\left(t_{1}^{-}\right)\right) \\
& +\int_{0}^{\omega}(\omega-s)^{\alpha-1} E_{\alpha, \alpha}\left[A(\omega-s)^{\alpha}\right] \\
& \times[A \varphi(0)+B x(s-\tau)] d s \\
& +\left[x_{\omega}-\varphi(0)-I_{1}\left(x\left(t_{1}^{-}\right)\right)\right. \\
& -\int_{0}^{\omega}(\omega-\theta)^{\alpha-1} E_{\alpha, \alpha}\left(A(\omega-\theta)^{\alpha}\right) \\
& \times(A \varphi(0)+B x(\theta-\tau)) d \theta]=x_{\omega} .
\end{aligned}
$$

Thus system (1) is controllable on $[0, \omega], \omega \in\left(t_{1}, t_{2}\right]$.
For $\omega \in\left(t_{i}, t_{i+1}\right], i=1,2, \ldots, k$, we take the control function as

$$
\begin{aligned}
u(t)= & C^{*}\left[E_{\alpha, \alpha}\left(A^{*}(\omega-t)^{\alpha}\right)\right] W_{c}^{-1}[0, \omega] \\
\times & {\left[x_{\omega}-\varphi(0)-\sum_{j=1}^{i} I_{j}\left(x\left(t_{j}^{-}\right)\right)\right.} \\
& -\int_{0}^{\omega}(\omega-\theta)^{\alpha-1} E_{\alpha, \alpha}\left(A(\omega-\theta)^{\alpha}\right) \\
& \times(A \varphi(0)+B x(\theta-\tau)) d \theta] .
\end{aligned}
$$

Substituting $t=\omega$ in (20) and inserting (30), then the same argument implies $x(\omega)=x_{\omega}$. Therefore system (1) is controllable on $[0, \omega]$.

Next, we prove necessity of Theorem 10. Suppose $W_{c}[0, \omega]$ is singular, without loss of generality; for $\omega \in\left(t_{i}, t_{i+1}\right], i=$ $1,2, \ldots, k$, there exists a nonzero vector $z_{0}$ such that

$$
z_{0}^{*} W_{c}[0, \omega] z_{0}=0
$$

That is,

$$
\begin{aligned}
& \int_{0}^{\omega} z_{0}^{*}(\omega-s)^{\alpha-1}\left[E_{\alpha, \alpha}\left(A(\omega-s)^{\alpha}\right)\right] \\
& \quad \times C C^{*}\left[E_{\alpha, \alpha}\left(A^{*}(\omega-s)^{\alpha}\right)\right] z_{0} d s=0 .
\end{aligned}
$$

Then it follows

$$
z_{0}^{*} E_{\alpha, \alpha}\left[A(\omega-s)^{\alpha}\right] C=0
$$

on $s \in[0, \omega]$. Since system (1) is controllable, there exist control inputs $u_{1}(t)$ and $u_{2}(t)$ such that

$$
\begin{aligned}
& x(\omega)= \varphi(0)+\sum_{j=1}^{i} I_{j}\left(x\left(t_{j}^{-}\right)\right) \\
&+ \int_{0}^{\omega}(\omega-s)^{\alpha-1} E_{\alpha, \alpha}\left[A(\omega-s)^{\alpha}\right] \\
& \times\left[A \varphi(0)+B x(s-\tau)+C u_{1}(s)\right] d s=0, \\
& z_{0}= \varphi(0)+\sum_{j=1}^{i} I_{j}\left(x\left(t_{j}^{-}\right)\right) \\
&+\int_{0}^{\omega}(\omega-s)^{\alpha-1} E_{\alpha, \alpha}\left[A(\omega-s)^{\alpha}\right] \\
& \quad \times\left[A \varphi(0)+B x(s-\tau)+C u_{2}(s)\right] d s .
\end{aligned}
$$

Combining (34) and (35) yields

$$
z_{0}-\int_{0}^{\omega}(\omega-s)^{\alpha-1} E_{\alpha, \alpha}\left[A(\omega-s)^{\alpha}\right] C\left[u_{2}(s)-u_{1}(s)\right] d s=0 .
$$


Multiplying $z_{0}^{*}$ on both sides of (36), we get

$$
\begin{gathered}
z_{0}^{*} z_{0}-\int_{0}^{\omega}(\omega-s)^{\alpha-1} z_{0}^{*} E_{\alpha, \alpha}\left[A(\omega-s)^{\alpha}\right] \\
\times C\left[u_{2}(s)-u_{1}(s)\right] d s=0 .
\end{gathered}
$$

According to $z_{0}^{*} E_{\alpha, \alpha}\left[A(\omega-s)^{\alpha}\right] C=0$, we have $z_{0}^{*} z_{0}=0$. Thus $z_{0}=0$. This contradiction therefore completes the proof.

Theorem 10 presents a geometric type criterion. By the algebraic transform and computation, we can obtain an algebraic criterion which is similar to the famous Kalman's rank condition [19].

Theorem 11. System (1) is controllable on $[0, \omega]$ if and only if

$$
\operatorname{rank}\left[C|A C| \cdots \mid A^{n-1} C\right]=n \text {. }
$$

Proof. According to Cayley-Hamilton theorem, $t^{\alpha-1} E_{\alpha, \alpha}\left(A t^{\alpha}\right)$ can be represented as

$$
t^{\alpha-1} E_{\alpha, \alpha}\left(A t^{\alpha}\right)=\sum_{k=0}^{+\infty} \frac{t^{k \alpha+\alpha-1}}{\Gamma(k \alpha+\alpha)} A^{k}=\sum_{k=0}^{n-1} G_{k}(t) A^{k} .
$$

For $\omega \in\left[0, t_{1}\right]$

$$
\begin{aligned}
& x(\omega)=\varphi(0)+\int_{0}^{\omega}(\omega-s)^{\alpha-1} E_{\alpha, \alpha}\left[A(\omega-s)^{\alpha}\right] \\
& \quad \times[A \varphi(0)+B x(s-\tau)+C u(s)] d s \\
&=\varphi(0)+\sum_{k=0}^{n-1} \int_{0}^{\omega} G_{k}(\omega-s) A^{k} \\
& \quad \times[A \varphi(0)+B x(s-\tau)+C u(s)] d s .
\end{aligned}
$$

Let

$$
\Psi=\varphi(0)+\sum_{k=0}^{n-1} \int_{0}^{\omega} G_{k}(\omega-s) A^{k}[A \varphi(0)+B x(s-\tau)] d s .
$$

Then combining (40) with (41) yields

$$
\begin{aligned}
x(\omega)-\Psi & =\sum_{k=0}^{n-1} A^{k} C \int_{0}^{\omega} G_{k}(\omega-s) u(s) d s \\
& =\left[C|A C| \cdots \mid A^{n-1} C\right]\left[\begin{array}{c}
d_{0} \\
d_{1} \\
\vdots \\
d_{n-1}
\end{array}\right],
\end{aligned}
$$

where $d_{k}=\int_{0}^{\omega} G_{k}(\omega-s) u(s) d s, k=0,1, \ldots, n-1$. Note that, for arbitrary $\varphi \in \mathbf{C}\left([-\tau, 0], \mathbb{R}^{n}\right)$ and $x(\omega) \in \mathbb{R}^{n}$, the sufficient and necessary condition to have a control input $u(t)$ satisfying (42) is that

$$
\operatorname{rank}\left[C|A C| \cdots \mid A^{n-1} C\right]=n
$$

For $\omega \in\left(t_{i}, t_{i+1}\right], \quad i=1,2, \ldots, k$,

$$
\begin{aligned}
x(\omega)= & \varphi(0)+\sum_{j=1}^{i} I_{j}\left(x\left(t_{j}^{-}\right)\right) \\
& +\int_{0}^{\omega}(\omega-s)^{\alpha-1} E_{\alpha, \alpha}\left[A(\omega-s)^{\alpha}\right] \\
& \times[A \varphi(0)+B x(s-\tau)+C u(s)] d s \\
= & \varphi(0)+\sum_{j=1}^{i} I_{j}\left(x\left(t_{j}^{-}\right)\right) \\
& +\sum_{k=0}^{n-1} \int_{0}^{\omega} G_{k}(\omega-s) A^{k}
\end{aligned}
$$$$
\times[A \varphi(0)+B x(s-\tau)+C u(s)] d s .
$$

Combining (41) with (44) yields

$$
\begin{aligned}
x(\omega)-\Psi-\sum_{j=1}^{i} I_{j}\left(x\left(t_{j}^{-}\right)\right) & =\sum_{k=0}^{n-1} A^{k} C \int_{0}^{\omega} G_{k}(\omega-s) u(s) d s \\
& =\left[C|A C| \cdots \mid A^{n-1} C\right]\left[\begin{array}{c}
d_{0} \\
d_{1} \\
\vdots \\
d_{n-1}
\end{array}\right] .
\end{aligned}
$$

Note that, for arbitrary $\varphi \in \mathbf{C}\left([-\tau, 0], \mathbb{R}^{n}\right)$ and $x(\omega) \in \mathbb{R}^{n}$, the sufficient and necessary condition to have a control input $u(t)$ satisfying $(45)$ is that

$$
\operatorname{rank}\left[C|A C| \cdots \mid A^{n-1} C\right]=n
$$

Thus, the proof is completed.

Remark 12. System (1) is controllable if and only if the resolvent condition $\lambda\left(\lambda I+Q_{\omega}\right)^{-1} \rightarrow 0$ as $\lambda \rightarrow 0$ holds (here $Q_{\omega}$ is the respective Gramian matrix in the nonfractional, nondelay, and nonimpulsive case) since this is equivalent to the rank condition in the finite dimensional case $[19,35,36]$.

\section{Illustrative Examples}

In this section, we give two examples to illustrate the presented criteria. 
Example 13. Consider the controllability of linear fractional differential systems with state delay and impulses as follows:

$$
\begin{aligned}
D^{1 / 2} x(t)= & {\left[\begin{array}{ll}
0 & 1 \\
0 & 0
\end{array}\right] x(t)+\left[\begin{array}{cc}
-1 & 2 \\
3 & 1
\end{array}\right] x\left(t-\frac{1}{3}\right) } \\
& +\left[\begin{array}{l}
2 \\
1
\end{array}\right] u(t), \quad t \in[0,4] \backslash\{1,2,3\}, \\
\Delta x\left(t_{i}\right) & =\frac{1}{2} x\left(t_{i}^{-}\right), \quad t_{i}=i, i=1,2,3, \\
x(t) & =e^{t}, \quad t \in\left[-\frac{1}{3}, 0\right] .
\end{aligned}
$$

Now, we apply Theorem 10 to prove that system (47) is controllable on $[0,4]$. Let us take

$$
\alpha=\frac{1}{2}, \quad A=\left[\begin{array}{ll}
0 & 1 \\
0 & 0
\end{array}\right], \quad B=\left[\begin{array}{cc}
-1 & 2 \\
3 & 1
\end{array}\right], \quad C=\left[\begin{array}{l}
2 \\
1
\end{array}\right] \text {. }
$$

By computation, we have

$$
\begin{aligned}
& C C^{*}=\left[\begin{array}{l}
2 \\
1
\end{array}\right]\left[\begin{array}{ll}
2 & 1
\end{array}\right]=\left[\begin{array}{ll}
4 & 2 \\
2 & 1
\end{array}\right], \\
& E_{1 / 2,1 / 2}\left(A(4-s)^{1 / 2}\right)= \sum_{k=0}^{1} \frac{A^{k}(4-s)^{k / 2}}{\Gamma(k / 2+1 / 2)} \\
&=\left[\begin{array}{cc}
\frac{1}{\sqrt{\pi}}(4-s)^{\frac{1}{2}} \\
0 & \frac{1}{\sqrt{\pi}}
\end{array}\right], \\
& E_{1 / 2,1 / 2}\left(A^{*}(4-s)^{1 / 2}\right)=\sum_{k=0}^{1} \frac{A^{k}(4-s)^{k / 2}}{\Gamma(k / 2+1 / 2)} \\
&=\left[\begin{array}{cc}
\frac{1}{\sqrt{\pi}} & 0 \\
(4-s)^{1 / 2} & \frac{1}{\sqrt{\pi}}
\end{array}\right] .
\end{aligned}
$$

Substituting $\omega=4$ in (25) and combining (25) with (49)-(51) yield

$$
\begin{aligned}
W_{c}[0,4]= & \int_{0}^{4}(4-s)^{-1 / 2}\left[E_{1 / 2,1 / 2}\left(A(4-s)^{1 / 2}\right)\right] \\
& \times C C^{*}\left[E_{1 / 2,1 / 2}\left(A^{*}(4-s)^{1 / 2}\right)\right] d s \\
= & {\left[\begin{array}{cc}
\frac{16}{\pi}+\frac{16}{\sqrt{\pi}}+\frac{16}{3} & \frac{8}{\pi}+\frac{4}{\sqrt{\pi}} \\
\frac{8}{\pi}+\frac{4}{\sqrt{\pi}} & \frac{4}{\pi}
\end{array}\right] . }
\end{aligned}
$$

Obviously, $W_{c}[0,4]$ is nonsingular. Thus by Theorem 10 , system (47) is controllable on $[0,4]$.
Example 14. Consider the controllability of linear fractional differential systems with state delay and impulses as follows:

$$
\begin{aligned}
& D^{1 / 3} x(t)= {\left[\begin{array}{ccc}
0 & 1 & 0 \\
0 & 0 & 1 \\
-2 & -4 & -3
\end{array}\right] x(t)+\left[\begin{array}{ccc}
1 & 0 & 1 \\
2 & 1 & 1 \\
2 & -1 & 0
\end{array}\right] x\left(t-\frac{\pi}{3}\right) } \\
&+ {\left[\begin{array}{cc}
1 & 0 \\
0 & 1 \\
-1 & 1
\end{array}\right] u(t), } \\
& \qquad t \in\left[0, \frac{5}{2} \pi\right] \backslash\left\{\frac{\pi}{2}, \pi, \frac{3}{2} \pi, 2 \pi\right\}, \\
& \Delta x\left(t_{i}\right)=\frac{1}{3} x\left(t_{i}^{-}\right), \quad t_{i}=\frac{i \pi}{2}, \quad i=1,2,3,4, \\
& x(t)=\sin t, \quad t \in\left[-\frac{\pi}{3}, 0\right] .
\end{aligned}
$$

Now, we apply Theorem 11 to prove that system (53) is controllable on $[0,(5 / 2) \pi]$. Let us take

$$
\begin{gathered}
\alpha=\frac{1}{3}, \quad A=\left[\begin{array}{ccc}
0 & 1 & 0 \\
0 & 0 & 1 \\
-2 & -4 & -3
\end{array}\right], \\
B=\left[\begin{array}{ccc}
1 & 0 & 1 \\
2 & 1 & 1 \\
2 & -1 & 0
\end{array}\right], \quad C=\left[\begin{array}{cc}
1 & 0 \\
0 & 1 \\
-1 & 1
\end{array}\right] .
\end{gathered}
$$

Then one can obtain

$$
\begin{aligned}
& \operatorname{rank} {[C|A C| \cdots \mid} \\
& \quad=\operatorname{rank}\left[\begin{array}{cccccc}
1 & 0 & 0 & \star & \star & \star \\
0 & 1 & -1 & \star & \star & \star \\
0 & 0 & 2 & \star & \star & \star
\end{array}\right]=3 .
\end{aligned}
$$

Thus by Theorem 11 , system $(53)$ is controllable on $[0,(5 / 2) \pi]$.

\section{Conclusions}

In this paper, the controllability criteria for linear fractional differential systems with delay in the state and impulses have been investigated. The sufficient and necessary conditions for the controllability of such systems have been established. Furthermore, both the proposed criteria and illustrative examples have shown that the controllability property of the linear systems is dependent neither on the order of fractional derivative, on delay nor on impulses.

\section{Acknowledgments}

The authors are very grateful to the Associate Editor, Professor Francisco J. Marcellán, and the two anonymous reviewers for their helpful and valuable comments and suggestions, which significantly contributed to improving the quality of this paper. This work is jointly supported by the National Natural Science Foundation of China under Grant nos. 
61272530 and 11072059 , the Natural Science Foundation of Jiangsu Province of China under Grant no. BK2012741, and the Key Program of Educational Commission of Anhui Province of China under Grant no. KJ2011A197.

\section{References}

[1] K. S. Miller and B. Ross, An Introduction to the Fractional Calculus and Fractional Differential Equations, John Wiley \& Sons, New York, NY, USA, 1993.

[2] I. Podlubny, Fractional Differential Equations, vol. 198 of Mathematics in Science and Engineering, Technical University of Kosice, Kosice, Slovak Republic, 1999.

[3] K. Diethelm, The Analysis of Fractional Differential Equations, Springer, Berlin, Germany, 2010.

[4] A. A. Kilbas, H. M. Srivastava, and J. J. Trujillo, Theory and Applications of Fractional Differential Equations, vol. 204 of North-Holland Mathematics Studies, Elsevier Science B.V., Amsterdam, The Netherlands, 2006.

[5] Z. M. Odibat, "Analytic study on linear systems of fractional differential equations," Computers \& Mathematics with Applications, vol. 59, no. 3, pp. 1171-1183, 2010.

[6] Y. Zhou and F. Jiao, "Nonlocal Cauchy problem for fractional evolution equations," Nonlinear Analysis. Real World Applications. An International Multidisciplinary Journal, vol. 11, no. 5, pp. 4465-4475, 2010.

[7] J. Wang and H. Xiang, "Upper and lower solutions method for a class of singular fractional boundary value problems with $p$ Laplacian operator," Abstract and Applied Analysis, vol. 2010, Article ID 971824, 12 pages, 2010.

[8] J. Deng and L. Ma, "Existence and uniqueness of solutions of initial value problems for nonlinear fractional differential equations," Applied Mathematics Letters, vol. 23, no. 6, pp. 676680, 2010.

[9] R. W. Ibrahim, "Complex transforms for systems of fractional differential equations," Abstract and Applied Analysis, vol. 2012, Article ID 814759, 15 pages, 2012.

[10] K. Sayevand, A. Golbabai, and A. Yildirim, "Analysis of differential equations of fractional order," Applied Mathematical Modelling, vol. 36, no. 9, pp. 4356-4364, 2012.

[11] W. Q. Wu and X. B. Zhou, "Eigenvalue of fractional differential equations with Laplacian operator," Discrete Dynamics in Nature and Society, vol. 2013, Article ID 137890, 8 pages, 2013.

[12] X. Zhang, "Some results of linear fractional order time-delay system," Applied Mathematics and Computation, vol. 197, no. 1, pp. 407-411, 2008.

[13] Y. Zhou, F. Jiao, and J. Li, "Existence and uniqueness for $p$-type fractional neutral differential equations," Nonlinear Analysis. Theory, Methods \& Applications. An International Multidisciplinary Journal. Series A: Theory and Methods, vol. 71, no. 7-8, pp. 2724-2733, 2009.

[14] F. Chen and Y. Zhou, "Attractivity of fractional functional differential equations," Computers \& Mathematics with Applications, vol. 62, no. 3, pp. 1359-1369, 2011.

[15] E. Kaslik and S. Sivasundaram, "Analytical and numerical methods for the stability analysis of linear fractional delay differential equations," Journal of Computational and Applied Mathematics, vol. 236, no. 16, pp. 4027-4041, 2012.

[16] D. Baĭnov and P. Simeonov, Impulsive Differential Equations: Periodic Solutions and Applications, John Wiley \& Sons, New York, NY, USA, 1993.
[17] M. Benchohra, J. Henderson, and S. Ntouyas, Impulsive Differential Equations and Inclusions, vol. 2, Hindawi Publishing Corporation, New York, NY, USA, 2006.

[18] M. Fečkan, Y. Zhou, and J. Wang, "On the concept and existence of solution for impulsive fractional differential equations," Communications in Nonlinear Science and Numerical Simulation, vol. 17, no. 7, pp. 3050-3060, 2012.

[19] R. E. Kalman, Y. C. Ho, and K. S. Narendra, "Controllability of linear dynamical systems," Contributions to Differential Equations, vol. 1, no. 2, pp. 189-213, 1963.

[20] W. M. Wonham and A. S. Morse, "Decoupling and pole assignment in linear multivariable systems: a geometric approach," SIAM Journal on Control and Optimization, vol. 8, pp. 1-18, 1970.

[21] R. Manzanilla, L. G. Mármol, and C. J. Vanegas, "On the controllability of a differential equation with delayed and advanced arguments," Abstract and Applied Analysis, vol. 2010, Article ID 307409, 16 pages, 2010.

[22] L. Wang, "Approximate boundary controllability for semilinear delay differential equations," Journal of Applied Mathematics, vol. 2011, Article ID 587890, 10 pages, 2011.

[23] H. Shi, G. Xie, and W. Luo, "Controllability analysis of linear discrete time systems with time delay in state," Abstract and Applied Analysis, vol. 2012, Article ID 10.1155/2012/490903, 11 pages, 2012.

[24] H. Shi, G. M. Xie, and W. G. Luo, "Controllability of linear discrete-time systems with both delayed states and delayed inputs," Abstract and Applied Analysis, vol. 2013, Article ID 975461, 5 pages, 2013.

[25] S. H. Chen, W. H. Ho, and J. H. Chou, "Robust local regularity and controllability of uncertain TS fuzzy descriptor systems," Journal of Applied Mathematics, vol. 2012, Article ID 825416, 14 pages, 2012.

[26] W. Jiang and W. Z. Song, "Controllability of singular systems with control delay," Automatica, vol. 37, no. 11, pp. 1873-1877, 2001.

[27] L. Zhang, Y. Ding, T. Wang, L. Hu, and K. Hao, "Controllability of second-order semilinear impulsive stochastic neutral functional evolution equations," Mathematical Problems in Engineering, vol. 2012, Article ID 748091, 13 pages, 2012.

[28] H. Shi and G. Xie, "Controllability and observability criteria for linear piecewise constant impulsive systems," Journal of Applied Mathematics, vol. 2012, Article ID 182040, 24 pages, 2012.

[29] X. J. Wan, Y. P. Zhang, and J. T. Sun, "Controllability of impulsive neutral functional differential inclusions in Banach spaces," Journal of Applied Mathematics, vol. 2013, Article ID 861568, 8 pages, 2013.

[30] S. A. Ammour, S. Djennoune, and M. Bettayeb, "A sliding mode control for linear fractional systems with input and state delays," Communications in Nonlinear Science and Numerical Simulation, vol. 14, no. 5, pp. 2310-2318, 2009.

[31] K. Balachandran, J. Y. Park, and J. J. Trujillo, "Controllability of nonlinear fractional dynamical systems," Nonlinear Analysis. Theory, Methods \& Applications. An International Multidisciplinary Journal. Series A: Theory and Methods, vol. 75, no. 4, pp. 1919-1926, 2012.

[32] K. Balachandran, J. Kokila, and J. J. Trujillo, "Relative controllability of fractional dynamical systems with multiple delays in control," Computers \& Mathematics with Applications, vol. 64, no. 10, pp. 3037-3045, 2012.

[33] A. Debbouche and D. Baleanu, "Exact null controllability for fractional nonlocal integrodifferential equations via implicit 
evolution system," Journal of Applied Mathematics, vol. 2012, Article ID 931975, 17 pages, 2012.

[34] N. I. Mahmudov, "Approximate controllability of fractional Sobolev-type evolution equations in Banach spaces," Abstract and Applied Analysis, vol. 2013, Article ID 502839, 9 pages, 2013.

[35] T. L. Guo, "Controllability and observability of impulsive fractional linear time-invariant system," Computers \& Mathematics with Applications, vol. 64, no. 10, pp. 3171-3182, 2012.

[36] X. F. Zhou, W. Jiang, and L. G. Hu, "Controllability of a fractional linear time-invariant neutral dynamical system," Applied Mathematics Letters, vol. 26, no. 4, pp. 418-424, 2013. 


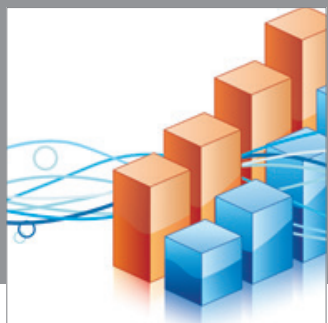

Advances in

Operations Research

mansans

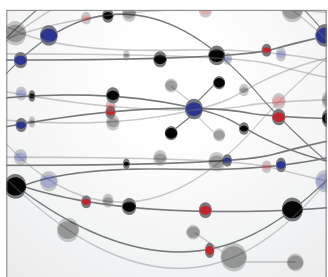

The Scientific World Journal
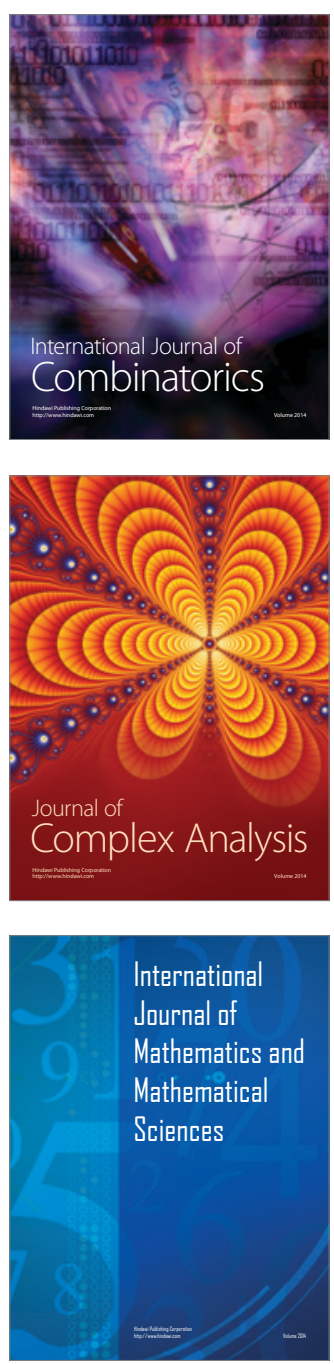
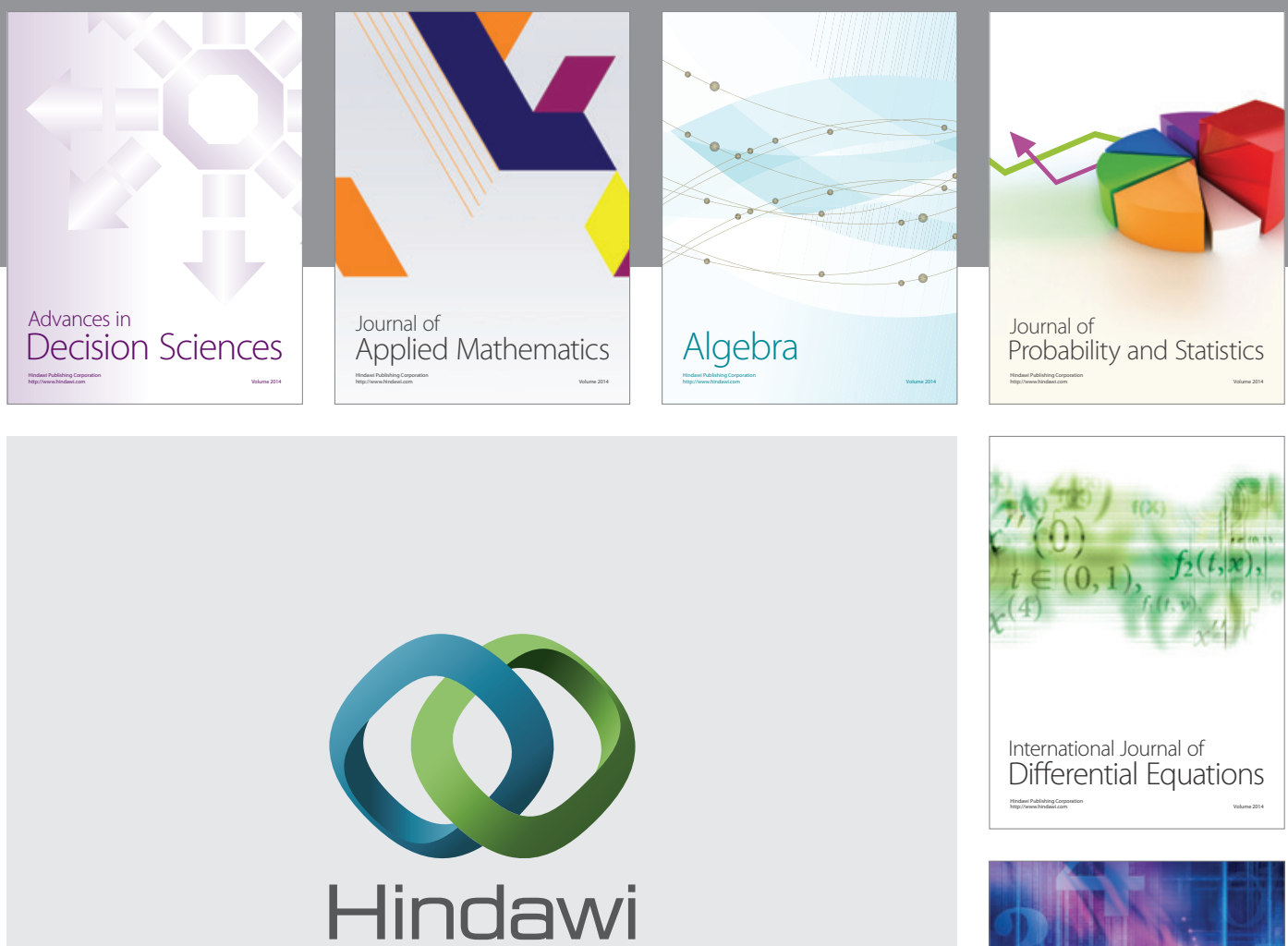

Submit your manuscripts at http://www.hindawi.com
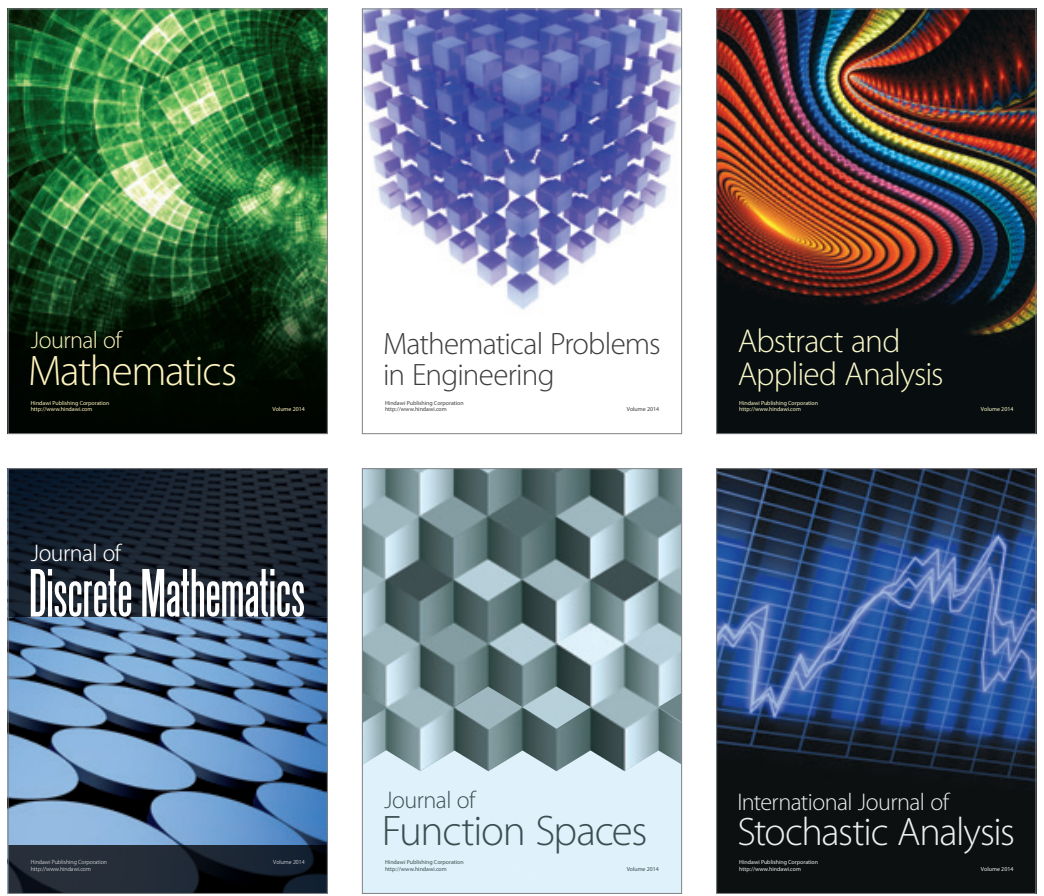

Journal of

Function Spaces

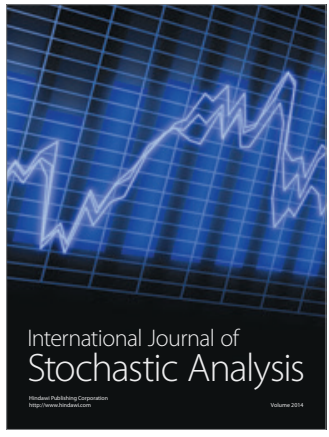

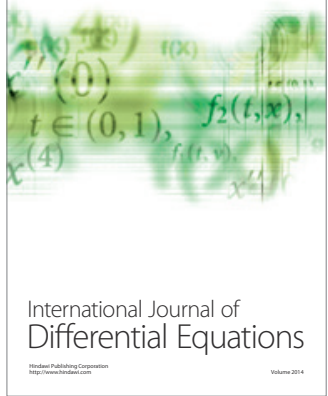
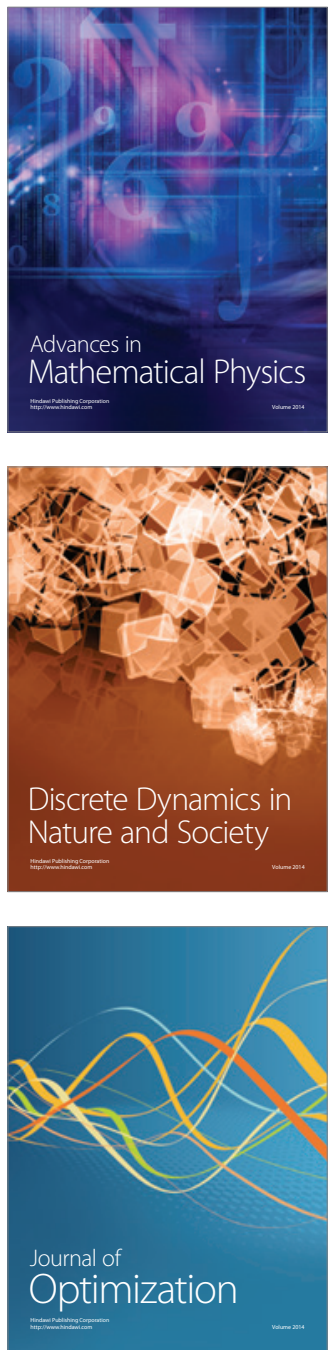